\title{
Light's Bending Angle in the Equatorial Plane of a Kerr Black Hole
}

\author{
S. V. Iyer* and E. C. Hansen \\ Department of Physics \& Astronomy, \\ State University of New York at Geneseo, \\ 1 College Circle, Geneseo, NY 14454.
}

\begin{abstract}
We present here a detailed derivation of an explicit spin-dependent expression for the bending angle of light as it traverses in the equatorial plane of a spinning black hole. We show that the deflection produced in the presence of the black hole angular momentum explicitly depends on whether the motion of the light ray is in the direction, or opposite to the spin. Compared to the zero-spin Schwarzschild case, the bending angle is greater for direct orbits, and smaller for retrograde orbits, confirming our physical intuition about the loss of left-right symmetry from a lensing perspective. In addition, we show that for higher spins, the effect is more pronounced resulting in tighter winding of direct orbits with respect to the axis of rotation, and a higher degree of unwinding of retro orbits. A direct consequence of this effect is a shift in image positions in strong gravitational lensing.
\end{abstract}

Keywords: gravitational lensing, bending angle, Kerr black holes

*Electronic address: iyer@geneseo.edu

${ }^{\dagger}$ Electronic address: ech3@geneseo.edu 


\section{INTRODUCTION}

The deflection of light as it traverses through curved spacetime remains one of the few available observational tools to quantitatively study the geometry surrounding a strong source of gravitation. The study of gravity's effect on light, or gravitational lensing, began with Einstein's prediction in 1913, and the subsequent confirmation by Eddington in 1919, of the bending angle of light in the relatively weak gravitational field near the sun. The need for detailed study of gravitational lensing in both the weak and strong deflection regimes has become more compelling as the precision of our observational tools have grown many-fold in the past few decades.

Bending angle calculations [1, 2, 3, 4] for Schwarzschild and Kerr geometries show that as we approach the depths of the gravitational potential, the bending angle exceeds $2 \pi$ indicating that multiple looping of a light ray around the center of attraction is possible (see for example, [5], page 678). In strong deflection gravitational lensing, images formed as a result of this are referred to as relativistic images. In order to study these images, we need analytical expressions for light deflection in exact form and sometimes as perturbative series expansions. In this paper, we present a detailed analysis of deflection of light in the equatorial plane of the spinning, or Kerr, black hole. Indeed, the program was started by Darwin[1], continued by Boyer and Lindquist[6], Chandrasekhar[7] and many others since. The key difference between our approach and earlier work is that we obtain an explicit expression for the bending angle for both direct and retrograde motion. Our final result for the bending angle is expressed explicitly in terms of the black hole mass $\mathrm{m}_{\bullet}=G M / c^{2}$ and its spin parameter $a=J / M c$, where $J / M$ is the angular momentum per unit mass of the black hole.

[8] for the Schwarzschild black hole and apply it to the case of deflection of rays confined to the Kerr equatorial plane. Bending angles, and therefore the position and magnification of images, depend crucially on whether the light ray is traversing in the same or opposite direction to the direction of rotation. In the Schwarzschild case [8], we expressed the bending angle in terms of the invariant impact parameter $b$, and in fact found it useful there to use the variable $b^{\prime}=1-b_{c} / b$, where $b_{c}=3 \sqrt{3}$ m was the Schwarzschild critical impact parameter. For the equatorial Kerr case, we introduce a new definition for $b^{\prime}$ that includes 
the non-zero spin of the black hole. We are able to define precisely the approach towards the critical impact parameter which is referred to as the strong deflection limit (SDL). The weak deflection limit (WDL), on the other hand, in both the Schwarzschild and the Kerr case are easily defined in terms of the impact parameter as the limit $b \rightarrow \infty$. The detailed SDL and WDL series are presented elsewhere [9].

In Section 2, we set up the basic framework with definitions of the variables and sign convention. We also discuss the meaning of a number of limiting values as we cross-check our results with the zero-spin (Schwarzschild) case. We obtain a formal exact expression for the bending angle in terms of elliptic integrals of the third kind. A plot of the bending angle as a function of the impact parameter from the critical value all the way to infinity is presented. We believe that this plot has not appeared in literature before.

The exact bending angle is plotted numerically for both cases to show a remarkable difference between the deflection of light on the direct side and the retro side. On the retro side, as the light ray traverses "upstream" it suffers a smaller deflection angle; smaller even than the Schwarzschild bending angle. This unravelling of the light ray as it tries to loop around against the spin direction supports our physical intuition. On the direct side, the bending angle is greater than the Schwarzschild value, resulting in a tighter looping of the light ray. Moreover, we show that as we vary the spin parameter $a$ from low to high values, relativistic images are shifted inward on the direct side, and outward on the retro side. We derive series expansions of the bending angle in the strong and weak deflection limits, to be

presented elsewhere [9], and show that the analytical results can be used to predict image positions and magnifications.

\section{THE EXACT BENDING ANGLE FOR KERR GEOMETRY}

We begin with the Kerr line element expressed in the Boyer-Lindquist coordinates (with $\theta=\pi / 2$ for the equatorial plane):

$$
\mathrm{d} s^{2}=g_{t t} \mathrm{~d} t^{2}+g_{r r} \mathrm{~d} r^{2}+g_{\phi \phi} \mathrm{d} \phi^{2}+2 g_{t \phi} \mathrm{d} t \mathrm{~d} \phi
$$


where

$$
\begin{array}{r}
g_{t t}(r)=-\left(1-\frac{2 \mathrm{~m}_{\bullet}}{r}\right) \\
g_{r r}(r)=\left(1-\frac{2 \mathrm{~m}_{\bullet}}{r}+\frac{a^{2}}{r^{2}}\right)^{-1} \\
g_{\phi \phi}(r)=r^{2}+a^{2}+\frac{2 \mathrm{~m}_{\bullet} a^{2}}{r} \\
g_{t \phi}(r)=\frac{-2 \mathrm{~m}_{\bullet} a}{r}
\end{array}
$$

where $t=c \tau$ and $\mathrm{m}_{\bullet}=G M / c^{2}$ (gravitational radius) with $\tau$ physical time and $M$ the physical mass of the black hole. From the Euler-Lagrange equations for null geodesics, we have

$$
\dot{r}^{2}=\left[\mathcal{E}^{2}+\frac{\mathcal{E}^{2} a^{2}}{r^{2}}+\frac{2 M \mathcal{E}^{2} a^{2}}{r^{3}}-\frac{4 M a \mathcal{E} J_{z}}{r^{3}}-\frac{J_{z}^{2}}{r^{2}}+\frac{2 M J_{z}^{2}}{r^{3}}\right]
$$

and

$$
\dot{\phi}=\frac{J_{z}\left[1-\frac{2 \mathrm{~m}_{\bullet}}{r}+\frac{2 \mathrm{~m}_{\bullet} a}{r} \frac{\mathcal{E}}{J_{z}}\right]}{\left(r^{2}-2 \mathrm{~m}_{\bullet} r+a^{2}\right)}
$$

Couched in the quantity $J_{z}$ that appears even at this early stage, is the sign of the angular momentum. This will decide whether the light ray is traversing along the direction of framedragging or opposite to it. We will use the following notation to keep track of the two types of orbits as we go through the analysis. We define

$$
b_{s}=s\left|\frac{J_{z}}{\mathcal{E}}\right| \equiv s b
$$

with $s=\operatorname{Sign}\left(J_{z} / \mathcal{E}\right)$ and $b$ is the positive magnitude. The parameter $s$ is positive for direct orbits and negative for retrograde orbits as shown in Figure 1.

With $a=J / M c$ it is convenient to introduce the following notation:

$$
\hat{a}=\frac{a}{m_{\bullet}}=\frac{J c}{G M^{2}}
$$

limiting ourselves to cases where $0 \leq \hat{a} \leq 1$, with $\hat{a}=0$ being the Schwarzschild limit and $\hat{a}=1$ being extreme Kerr. Orbits with $s=+1$ and $b_{s}>0$ will be referred to as direct or prograde orbits; and those with $s=-1$ and $b_{s}<0$ as retrograde orbits.

Rewriting the above expression, we have

$$
\dot{r}^{2}=J_{z}^{2}\left[\frac{1}{b^{2}}+\frac{a^{2}}{b^{2} r^{2}}+\frac{2 \mathrm{~m}_{\bullet} a^{2}}{r^{3} b^{2}}-\frac{4 \mathrm{~m}_{\bullet} a}{r^{3} b_{s}}-\frac{1}{r^{2}}+\frac{2 \mathrm{~m}_{\bullet}}{r^{3}}\right]
$$




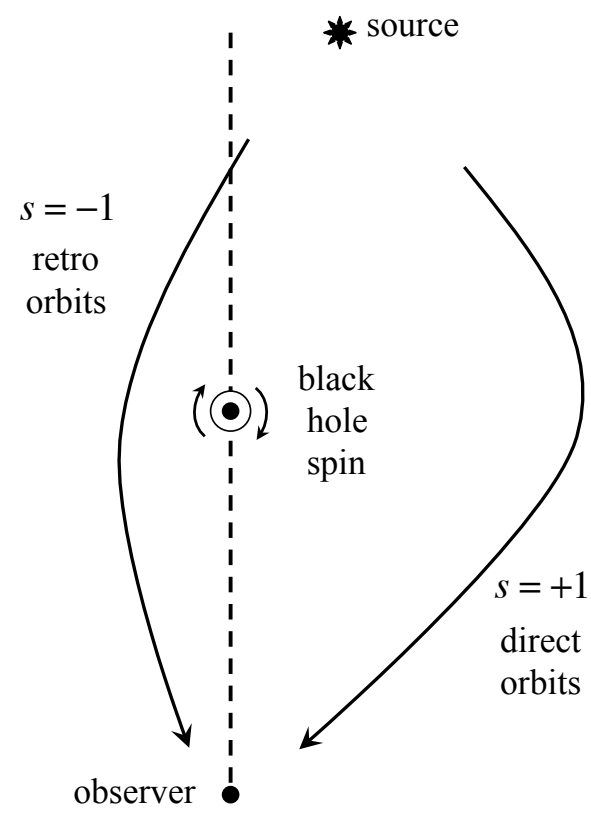

FIG. 1: Sign convention for orbits as viewed from above. The spin axis points into the page in this figure.

Next, letting $u=1 / r$ and using

$$
\left(\frac{d u}{d \phi}\right)^{2}=\frac{u^{4}(\dot{r})^{2}}{(\dot{\phi})^{2}}
$$

we have

$$
(\dot{\phi})^{2}=J_{z}^{2}\left[\frac{1-2 \mathrm{~m}_{\bullet} u+2 \mathrm{~m}_{\bullet} a u / b_{s}}{a^{2}-\left(2 \mathrm{~m}_{\bullet} / u\right)+\left(1 / u^{2}\right)}\right]^{2}
$$

and

$$
(\dot{r})^{2}=J_{z}^{2}\left[\frac{1}{b^{2}}+2 \mathrm{~m}_{\bullet} u^{3}\left(1-\frac{a}{b_{s}}\right)^{2}-u^{2}\left(1-\frac{a^{2}}{b^{2}}\right)\right] .
$$

Combining the above expressions and simplifying gives us

$$
\left(\frac{d u}{d \phi}\right)^{2}=\frac{\left[1-2 \mathrm{~m}_{\bullet} u+a^{2} u^{2}\right]^{2}}{\left[1-2 \mathrm{~m}_{\bullet} u\left(1-a / b_{s}\right)\right]^{2}} B(u),
$$

where the quantity $B(u)$, a cubic polynomial given by

$$
B(u)=\left[2 \mathrm{~m} \cdot\left(1-\frac{a}{b_{s}}\right)^{2} u^{3}-\left(1-\frac{a^{2}}{b^{2}}\right) u^{2}+\frac{1}{b^{2}}\right]
$$

has a maximum of two positive roots and at most one negative root. We consider the case of one real negative root $u_{1}$ and two real distinct positive roots $u_{2}$ and $u_{3}$. The three roots, 
given in terms of two intermediate constants $P$ and $Q$ that allow us to line up the roots in the order $u_{1}<u_{2}<u_{3}$ are given by

$$
\begin{aligned}
& u_{1}=\frac{P-2 \mathrm{~m}_{\bullet}-Q}{4 \mathrm{~m}_{\bullet} r_{0}} \\
& u_{2}=\frac{1}{r_{0}} \\
& u_{3}=\frac{P-2 \mathrm{~m}_{\bullet}+Q}{4 \mathrm{~m}_{\bullet} r_{0}}
\end{aligned}
$$

By comparing the coefficients in $B(u)$ to those in the original polynomial in equation(12) we first obtain the following relationship between $P$ and $\left\{a, b, s, r_{0}\right\}$

$$
P=r_{0} \frac{\left(1-\frac{a^{2}}{b^{2}}\right)}{\left(1+\frac{a}{b_{s}}\right)^{2}}=r_{0} \frac{\left(1+\frac{a}{b_{s}}\right)}{\left(1-\frac{a}{b_{s}}\right)}
$$

where $a$ and $b$ are positive quantities. This gives us the following relation between the critical parameters:

$$
r_{s c}=3 \mathrm{~m} \cdot \frac{\left(1-\frac{a}{b_{s c}}\right)}{\left(1+\frac{a}{b_{s c}}\right)} .
$$

Note that we need to set $r_{0}=r_{s c}, b_{s}=b_{s c}$ and $P=3 \mathrm{~m}$. to obtain the critical values. Note that when $a=0, r_{s c} \rightarrow r_{c}=3 \mathrm{~m}_{\bullet}$ in both cases. The critical value for $r_{0}$ is less than $3 \mathrm{~m}_{\bullet}$ for direct orbits and greater than $3 \mathrm{~m}$. for retro orbits.

Comparing the other coefficients of the cubic polynomial $B(u)$ we obtain the following additional expressions:

$$
\begin{array}{r}
\frac{Q^{2}-\left(P-2 \mathrm{~m}_{\bullet}\right)^{2}}{8 \mathrm{~m}_{\bullet} r_{0}^{3}}=\frac{1}{b^{2}\left(1-a / b_{s}\right)^{2}} \\
Q^{2}=\left(P-2 \mathrm{~m}_{\bullet}\right)\left(P+6 \mathrm{~m}_{\bullet}\right) .
\end{array}
$$

The intermediate variables $Q$ and $P$ can be eliminated by combining the above relations to yield a simple cubic equation involving the impact parameter and the distance of closest 
approach given by

$$
r_{0}^{3}-b^{2} \frac{\left(1-\frac{a}{b_{s}}\right)^{3}}{\left(1+\frac{a}{b_{s}}\right)} r_{0}+2 \mathrm{~m}_{\bullet} b^{2}\left(1-\frac{a}{b_{s}}\right)^{2}=0 .
$$

which can be solved to yield

$$
r_{0}=\frac{2 b}{\sqrt{3}} \sqrt{1-\frac{a^{2}}{b^{2}}} \cos \left[\frac{1}{3} \cos ^{-1}\left(\frac{-3 \sqrt{3} \mathrm{~m}_{\bullet}}{b} \frac{\left(1-\frac{a}{b_{s}}\right)^{2}}{\left(1-\frac{a^{2}}{b^{2}}\right)^{3 / 2}}\right)\right]
$$

This expression for the distance of closest approach and the invariant impact parameter becomes important in both the SDL and WDL series expansions [9] completely in terms of the invariant normalized quantity $b^{\prime}$. Also, note that this expression reduces to its Schwarzschild version (see e.g., equation (9) in [8]) when we set the spin parameter to zero.

So, in the strong deflection limit, we have $P=3 \mathrm{~m}$. and the following expressions involving the critical quantities:

$$
r_{s c}=3 \mathrm{~m} \cdot \frac{\left(1-\frac{a}{b_{s c}}\right)}{\left(1+\frac{a}{b_{s c}}\right)}
$$

and

$$
\left(b_{s c}+a\right)^{3}=27 \mathrm{~m}_{\bullet}^{2}\left(b_{s c}-a\right) .
$$

Equations (21) and (22) agree with Chandrasekhar (eqn 82, p329).

Combining these two equations gives us the following relationship between the critical values

$$
b_{s c}^{2}=3 r_{s c}^{2}+a^{2}
$$

To solve the cubic equation (22) we need to consider the direct and retrograde motion separately. For direct orbits (i.e., $s=+1$ ) we have

$$
\left(b_{+c}+a\right)^{3}-27 \mathrm{~m}_{\bullet}^{2}\left(b_{+c}+a\right)+54 a \mathrm{~m}_{\bullet}^{2}=0
$$

the solution of which is given by

$$
b_{+c}=-a+6 \mathrm{~m}_{\bullet} \cos \left[\frac{1}{3} \cos ^{-1}\left(\frac{-a}{\mathrm{~m}_{\bullet}}\right)\right] .
$$




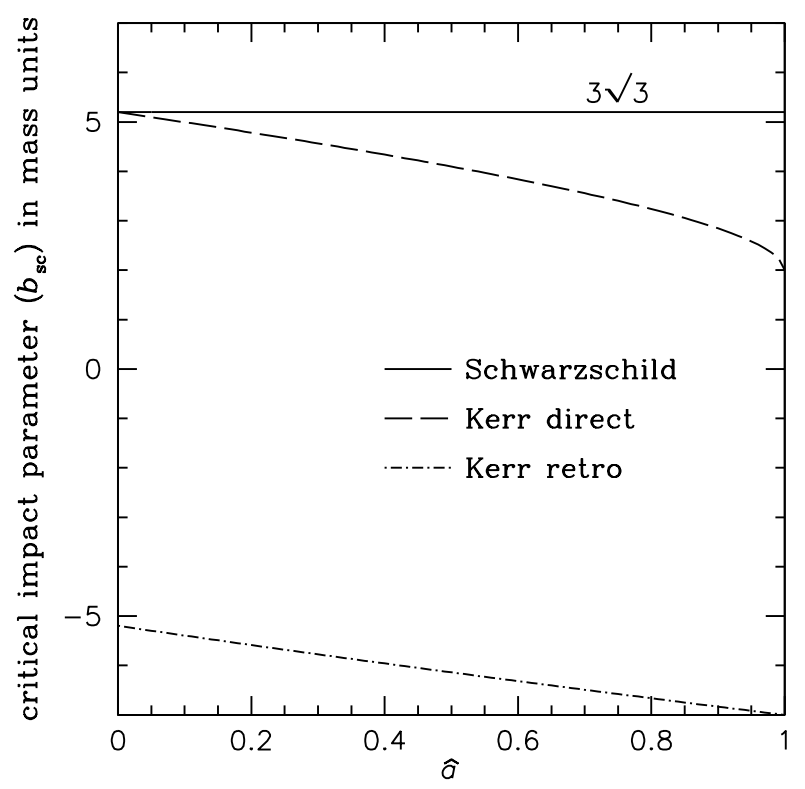

FIG. 2: The critical impact parameter in units of $\mathrm{m}_{\bullet}$ as a function of the spin parameter $\hat{a}$. The Schwarzschild critical impact parameter is shown for reference.

For retrograde orbits with $s=-1$ we have

$$
\left(b_{-c}-a\right)^{3}-27 \mathrm{~m}_{\bullet}^{2}\left(b_{-c}-a\right)-54 a \mathrm{~m}_{\bullet}^{2}=0
$$

Solving this cubic equation yields

$$
b_{-c}=-a-6 \mathrm{~m}_{\bullet} \cos \left[\frac{1}{3} \cos ^{-1}\left(\frac{a}{\mathrm{~m}_{\bullet}}\right)\right] .
$$

We can combine the two cases and write

$$
b_{s c}=-a+s 6 \mathrm{~m}_{\bullet} \cos \left[\frac{1}{3} \cos ^{-1}\left(\frac{-s a}{\mathrm{~m}_{\bullet}}\right)\right]
$$

Note that the critical impact parameter is a function of the black hole spin. In Figure 2, we have plotted the critical impact parameter as a function of $a$. From equation (22), we have the following:

$$
\begin{aligned}
\frac{\left(b_{s c}-a\right)}{\left(b_{s c}+a\right)} & =\frac{\left(b_{s c}+a\right)^{2}}{27 \mathrm{~m}_{\bullet}^{2}} \\
& =\frac{1}{27 \mathrm{~m}_{\bullet}^{2}}\left\{s 6 \mathrm{~m}_{\bullet} \cos \left[\frac{1}{3} \cos ^{-1}\left(\frac{-s a}{\mathrm{~m}_{\bullet}}\right)\right]\right\}^{2} \\
& =\frac{36}{27} \cos ^{2}\left[\frac{1}{3} \cos ^{-1}\left(\frac{-s a}{\mathrm{~m}_{\bullet}}\right)\right]
\end{aligned}
$$


Using the above, we obtain the following expression for the critical value of the distance of closest approach:

$$
r_{s c}=2 \mathrm{~m}_{\bullet}\left\{1+\cos \left[\frac{2}{3} \cos ^{-1}\left(\frac{-s a}{\mathrm{~m}_{\bullet}}\right)\right]\right\}
$$

which agrees with equation 87 on page 330 in [7]. Figure 3] is a plot of $r_{s c} / \mathrm{m}_{\bullet}$ as a function of spin. For $0 \leq a \leq \mathrm{m}_{\bullet}$, the critical value of $r_{0}$ lies in the following ranges: $\mathrm{m}_{\mathbf{0}} \leq r_{s c} \leq 3 \mathrm{~m}_{\mathbf{0}}$ for direct orbits and $3 \mathrm{~m}_{\bullet} \geq r_{s c} \geq 4 \mathrm{~m}_{\bullet}$ for retro orbits. Note that $r_{s c}$ stays positive throughout, given that this is the radial coordinate.

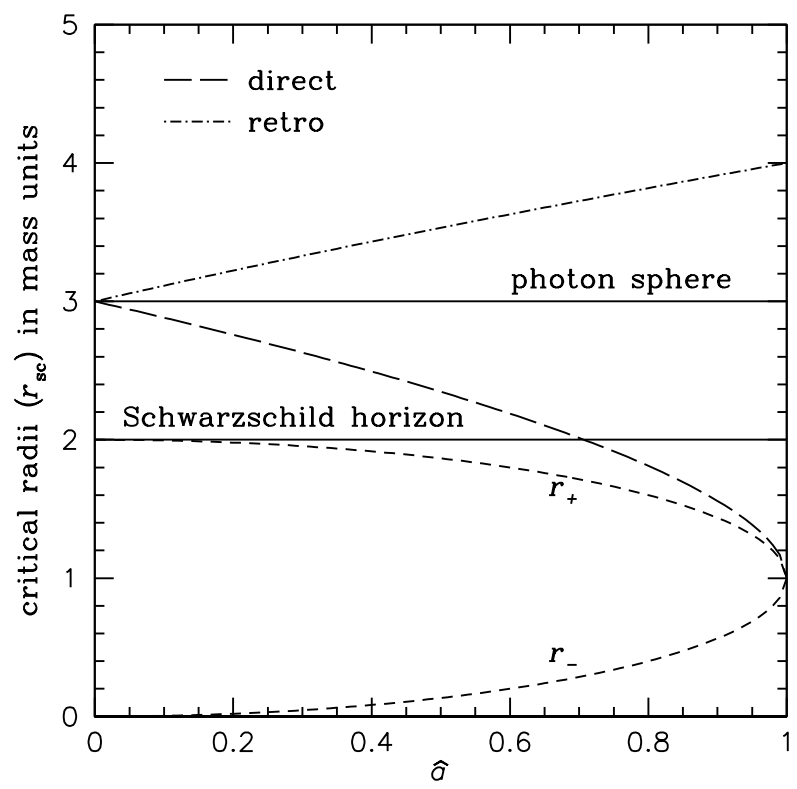

FIG. 3: The critical radius in units of $\mathrm{m}$. plotted as a function of the spin parameter $\hat{a}$. The Schwarzschild radius, the radius of the photon sphere and the Kerr inner and outer horizon radii are shown for reference.

In the extreme case when $a=M$, we have the following results:

$$
\begin{array}{rlll}
\text { direct: } & s=+1 & r_{+c}=\mathrm{m}_{\bullet} & b_{+c}=2 \mathrm{~m} \bullet \\
\text { retrograde: } & s=-1 & r_{-c}=4 \mathrm{~m}_{\bullet} & b_{-c}=-7 \mathrm{~m} .
\end{array}
$$

in agreement with equation 89 on page 330 in [7].

The sign of the impact parameter carries the information about whether the light propagation is in the same or opposite direction to the spin. This appears to be the most important 
difference in our approach, one that yields results pertaining to retrograde motion in a natural way. We believe that details involving retrograde motion are harder to extract when the sign of $a$ is used to keep track of the change in the direction of the black hole spin.

There are two other polynomials that appear in equation (11). We will denote the roots of $1-2 \mathrm{~m}_{\bullet} u+a^{2} u^{2}=0$ as $u_{ \pm}$, given by

$$
u_{ \pm}=\frac{m_{\bullet} \pm \sqrt{m_{\bullet}^{2}-a^{2}}}{a^{2}}
$$

Using this notation we can now write the ratio of the polynomials in terms of partial fractions as

$$
\frac{1-2 m_{\bullet} u\left(1-\omega_{s}\right)}{1-2 m_{\bullet} u+a^{2} u^{2}}=\frac{C_{+}}{\left(u_{+}-u\right)}+\frac{C_{-}}{\left(u_{-}-u\right)}
$$

Solving for $C_{+}$and $C_{-}$, we obtain

$$
C_{+}=\frac{\left(\mathrm{m}_{\bullet}+\sqrt{\mathrm{m}_{\bullet}^{2}-a^{2}}\right) 2 \mathrm{~m}_{\bullet}\left(1-\omega_{s}\right)-a^{2}}{2 a^{2} \sqrt{\mathrm{m}_{\bullet}^{2}-a^{2}}}
$$

and

$$
C_{-}=\frac{a^{2}-\left(\mathrm{m}_{\bullet}-\sqrt{\mathrm{m}_{\bullet}^{2}-a^{2}}\right) 2 \mathrm{~m}_{\bullet}\left(1-\omega_{s}\right)}{2 a^{2} \sqrt{\mathrm{m}_{\bullet}^{2}-a^{2}}}
$$

Let us now consider a light ray that starts in the asymptotic region and approaches the black hole, with $r_{0}$ as the distance of closest approach. It then emerges and reaches an observer who is also in an asymptotic region. Although the change in the $\phi$ coordinate of the light ray appears in the equations of motion, we will consider the deflection of the light ray from its original path $\hat{\alpha}$. The change in $\phi$ and the bending angle $\hat{\alpha}$ are simply related by a difference of $\pi$. The bending angle can be written down in a straightforward manner by integrating the equation of motion for $\phi$ and subtracting $\pi$ from it. The integrals therein can be separated into two parts as shown below to make the lower limit equal to the smallest root $u_{1}$. The integrals are then readily expressed in terms of elliptic integrals of the third kind (see [10], for example) to obtain the exact expression for the bending angle. These steps are shown below. 


$$
\begin{aligned}
& \hat{\alpha}=-\pi+\sqrt{\frac{2}{\mathrm{~m}_{\bullet}}} \frac{C_{+}}{1-\omega_{s}} \int_{0}^{1 / r_{0}} \frac{d u}{\left(u_{+}-u\right) \sqrt{\left(u-u_{1}\right)\left(u-u_{2}\right)\left(u-u_{3}\right)}} \\
& +\sqrt{\frac{2}{\mathrm{~m}_{\bullet}}} \frac{C_{-}}{1-\omega_{s}} \int_{0}^{1 / r_{0}} \frac{d u}{\left(u_{-}-u\right) \sqrt{\left(u-u_{1}\right)\left(u-u_{2}\right)\left(u-u_{3}\right)}} \\
& =-\pi+\sqrt{\frac{2}{m_{\bullet}}} \frac{C_{+}}{1-\omega_{s}}\left[\int_{u_{1}}^{u_{2}} \frac{d u}{\left(u_{+}-u\right) \sqrt{\left(u-u_{1}\right)\left(u_{2}-u\right)\left(u_{3}-u\right)}}\right. \\
& \left.-\int_{u_{1}}^{0} \frac{d u}{\left(u_{+}-u\right) \sqrt{\left(u-u_{1}\right)\left(u_{2}-u\right)\left(u_{3}-u\right)}}\right] \\
& +\sqrt{\frac{2}{\mathrm{~m}_{\mathbf{0}}}} \frac{C_{-}}{1-\omega_{s}}\left[\int_{u_{1}}^{u_{2}} \frac{d u}{\left(u_{-}-u\right) \sqrt{\left(u-u_{1}\right)\left(u_{2}-u\right)\left(u_{3}-u\right)}}\right. \\
& \left.-\int_{u_{1}}^{0} \frac{d u}{\left(u_{-}-u\right) \sqrt{\left(u-u_{1}\right)\left(u_{2}-u\right)\left(u_{3}-u\right)}}\right] \\
& \hat{\alpha}=-\pi+\frac{4}{1-\omega_{s}} \sqrt{\frac{r_{0}}{Q}}\left\{\Omega_{+}\left[\Pi\left(n_{+}, k\right)-\Pi\left(n_{+}, \psi, k\right)\right]+\Omega_{-}\left[\Pi\left(n_{-}, k\right)-\Pi\left(n_{-}, \psi, k\right)\right]\right\},
\end{aligned}
$$

where $\Pi\left(n_{ \pm}, k\right)$ and $\Pi\left(n_{ \pm}, \psi, k\right)$ are the complete and the incomplete elliptic integrals of the third kind respectively. The argument $k^{2}$ is defined through the elliptic integral as usual in the range $0 \leq k^{2} \leq 1$. Note that in some references the variable is referred to as $k^{2}$ and in others simply as $k$. The order in which the arguments appear in $\Pi(n, \psi, k)$ also varies between different references and in Mathematica.

Remark: In Mathematica, the built-in mathematical function for the incomplete elliptic integral of the third kind EllipticPi $[\mathrm{n}, \phi, \mathrm{m}]$ is defined by

$$
\int_{0}^{\phi}\left[1-\mathrm{n} \sin ^{2} \theta\right]^{-1}\left[1-\mathrm{m} \sin ^{2} \theta\right]^{-1 / 2} d \theta
$$

and the complete elliptic integral of the third kind is EllipticPi $[\mathrm{n}, \mathrm{m}]=\operatorname{EllipticPi}[\mathrm{n}, \pi / 2, \mathrm{~m}]$.

The other variables in the above expression are defined as follows:

$$
\begin{aligned}
\Omega_{ \pm} & =\frac{C_{ \pm}}{u_{ \pm}-u_{1}} \\
k^{2} & =\frac{Q-P+6 \mathrm{~m}_{\bullet}}{2 Q} \\
\psi & =\arcsin \sqrt{\frac{Q+2 \mathrm{~m}_{\bullet}-P}{Q+6 \mathrm{~m}_{\bullet}-P}} \\
n_{ \pm} & =\frac{u_{2}-u_{1}}{u_{ \pm}-u_{1}}
\end{aligned}
$$


It can be easily shown that in the limiting case when $a \rightarrow 0$, we have $\Omega_{+}=1, \Omega_{-}=0$ and $n_{+}=0$ to give

$$
\begin{aligned}
\hat{\alpha} & =-\pi+4 \sqrt{\frac{r_{0}}{Q}}[\Pi(0, k)-\Pi(\psi, 0, k)] \\
& =-\pi+4 \sqrt{\frac{r_{0}}{Q}}[K(k)-F(\psi, k)],
\end{aligned}
$$

where $K(k)$ and $F(\psi, k)$ are the complete and incomplete elliptic integrals of the first kind respectively. In addition, in the limit when $\mathrm{m}_{\bullet}=0$ (i.e., $h \rightarrow 0$ ) we recover zero deflection as expected.

We will use the following convenient notation:

$$
h=\frac{\mathrm{m}_{\bullet}}{r_{0}} \quad \omega_{s}=\frac{a}{b_{s}} \quad \text { and } \quad \omega_{0}=\frac{a^{2}}{\mathrm{~m}_{\bullet}^{2}}
$$

with $\omega_{s}$ taking on the appropriate sign for direct and retrograde orbit. Note that in the limit $\left\{\omega_{s}, \omega_{0} \rightarrow 0\right\}$, we recover the zero-spin Schwarzschild case, and in the limit $h \rightarrow 0$, we have the zero-deflection flat metric limit. Further, we define critical parameters analogous to the Schwarzschild case in [8]:

$$
h_{s c}=\frac{1+\omega_{s}}{1-\omega_{s}} \quad \text { and } \quad r_{s c}=\frac{3 \mathrm{~m}_{\bullet}}{h_{s c}}
$$

We also define the variable

$$
h^{\prime}=1-\frac{3 h}{h_{s c}} \equiv 1-3\left(\frac{\mathrm{m}_{\bullet}}{r_{0}}\right)\left(\frac{1-\omega_{s}}{1+\omega_{s}}\right)
$$

with

$$
1-3\left(\frac{\mathrm{m}_{\bullet}}{r_{0}}\right)\left(\frac{1-\omega_{s}}{1+\omega_{s}}\right) \stackrel{a \rightarrow 0}{\longrightarrow} 1-\frac{3 \mathrm{~m}_{\bullet}}{r_{0}} .
$$

We have introduced these different quantities for the Kerr case, keeping in mind that they should go over to those defined in the Schwarzschild case smoothly when $a$ is set equal to zero. So, as shown above, as $a \rightarrow 0, h_{s c} \rightarrow 1$ and we recover the definition of $h^{\prime}$ in [8]. In both cases, $h \rightarrow 0$ at critical, and $h \rightarrow 1$ as $r_{0}$ approaches infinity.

From a lensing perspective, we are interested in impact parameters beyond the critical value (SDL) extending all the way to infinity (WDL). We define the dimensionless quantity $b^{\prime}$ as

$$
b^{\prime}=1-\frac{s b_{s c}}{b}
$$


where the insertion of the quantity $s$ guarantees that the $b^{\prime}$ stays between 0 and 1 . Note that this definition goes over naturally in the Schwarzschild limit:

$$
1-\frac{s b_{s c}}{b} \stackrel{a \rightarrow 0}{\longrightarrow} 1-\frac{3 \sqrt{3} \mathrm{~m}_{\bullet}}{b}
$$

Now, some of the intermediate variables can be eliminated to rewrite all quantities in terms of $h, h_{s c}, \omega_{0}$ and $\omega_{s}$ as follows:

$$
\begin{aligned}
& \frac{r_{0}}{Q}=\frac{1}{h_{s c} \sqrt{\left(1-\frac{2 h}{h_{s c}}\right)\left(1+\frac{6 h}{h_{s c}}\right)}} \\
& k^{2}=\frac{\sqrt{\left(1-\frac{2 h}{h_{s c}}\right)\left(1+\frac{6 h}{h_{s c}}\right)}+\frac{6 h}{h_{s c}}-1}{2 \sqrt{\left(1-\frac{2 h}{h_{s c}}\right)\left(1+\frac{6 h}{h_{s c}}\right)}} \\
& \psi=\arcsin \sqrt{\frac{1-\frac{2 h}{h_{s c}}-\sqrt{\left(1-\frac{2 h}{h_{s c}}\right)\left(1+\frac{6 h}{h_{s c}}\right)}}{1-\frac{6 h}{h_{s c}}-\sqrt{\left(1-\frac{2 h}{h_{s c}}\right)\left(1+\frac{6 h}{h_{s c}}\right)}}} \\
& \Omega_{ \pm}=\frac{ \pm\left(1 \pm \sqrt{1-\omega_{0}}\right)\left(1-\omega_{s}\right) \mp \omega_{0} / 2}{\sqrt{1-\omega_{0}}\left(1 \pm \sqrt{1-\omega_{0}}-\frac{\omega_{0} h_{s c}}{4}\left[1-\frac{2 h}{h_{s c}}-\sqrt{\left.\left(1-\frac{2 h}{h_{s c}}\right)\left(1+\frac{6 h}{h_{s c}}\right)\right]}\right)\right.} \\
& n_{ \pm}=\frac{1-\frac{6 h}{h_{s c}}-\sqrt{\left(1-\frac{2 h}{h_{s c}}\right)\left(1+\frac{6 h}{h_{s c}}\right)}}{1-\frac{2 h}{h_{s c}}-\sqrt{\left(1-\frac{2 h}{h_{s c}}\right)\left(1+\frac{6 h}{h_{s c}}\right)}-\frac{4}{\omega_{0} h_{s c}}\left(1 \pm \sqrt{1-\omega_{0}}\right)}
\end{aligned}
$$

All of the above variables are to be substituted into the bending angle expression. We note here that the quantities $r_{0}, h, h_{s c}$, and $\omega_{s}$ depend on $b$, while $\omega_{0}=a^{2} / \mathrm{m}_{\bullet}^{2}$ is independent of $b$. Any quantity that has an " $s$ " in the subscript takes on a negative sign for retro orbits. The bending angle itself stays positive since the sign of $\phi$ in the equations of motion is determined by the incident ray in the asymptotic region. In other words, as the ray approaches critical on the retro side, the overall deflection is still towards the black hole even though the extent to which it is bent is smaller than in the static case. Another way of looking at this is to 
change the direction of the rotation axis from "into the page" in Figure 1 to "out of page" and look at the ray on the right side of the figure for which $s=-1$ now; the bending angle is still positive.

We now have an explicit expression for the bending angle (34) via equations (40)-(44) in terms of $b^{\prime}, a$ and $\mathrm{m}_{\bullet}$, the remaining independent quantities. In Figures 4 and 5 , we have plotted the exact bending angle as a function of $b^{\prime}$ for $\hat{a}=0.5$ and $\hat{a}=0.99$. Recall once again that the critical impact parameter depends on the spin parameter as well as on whether the light ray is undergoing direct or retrograde motion. As can be seen clearly from the bending angle plots, the bending angle is greater than the Schwarzschild value for direct orbits, and smaller for retro orbits. In addition for the higher spin $(\hat{a}=0.99)$ case the effect is much more pronounced. Plots for other values of $\hat{a}$ look similar to this, and in the limit $\hat{a}=0$, all three curves merge as expected.

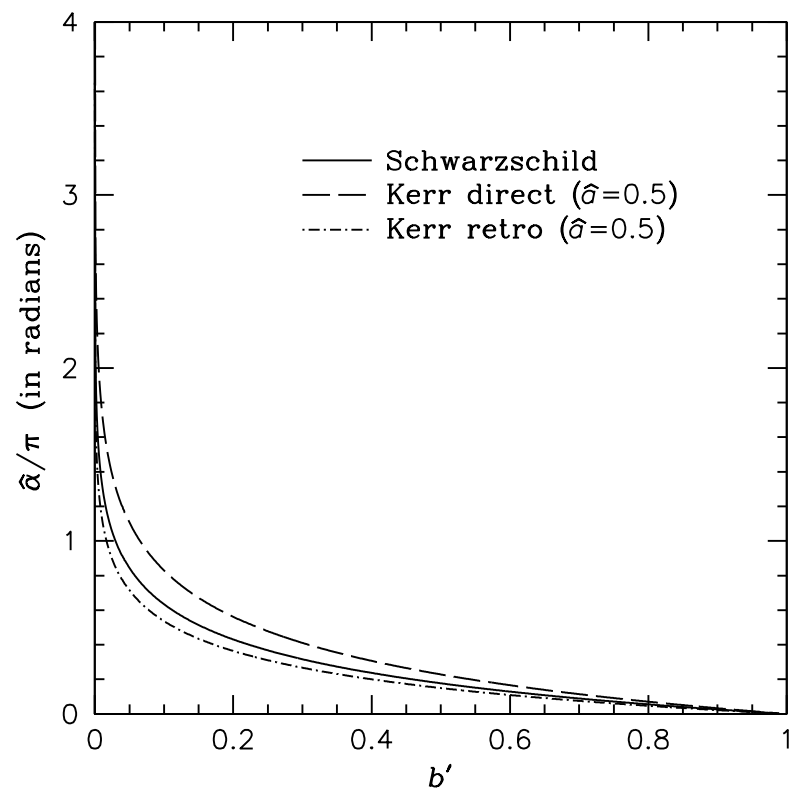

FIG. 4: Exact bending angle plotted as a function of the normalized impact parameter. The strong deflection regime is towards the left of this plot as $b^{\prime}$ goes to zero, and the weak deflection regime is as $b^{\prime} \rightarrow \infty$. The Schwarzschild bending angle is shown for reference.

One of the first remarkable features is the suppression of the bending angle on the retro side compared to the Schwarzschild case. On the direct side, light rays are bent more because of they are being swirled "downstream" with the spin, while motion of light rays 


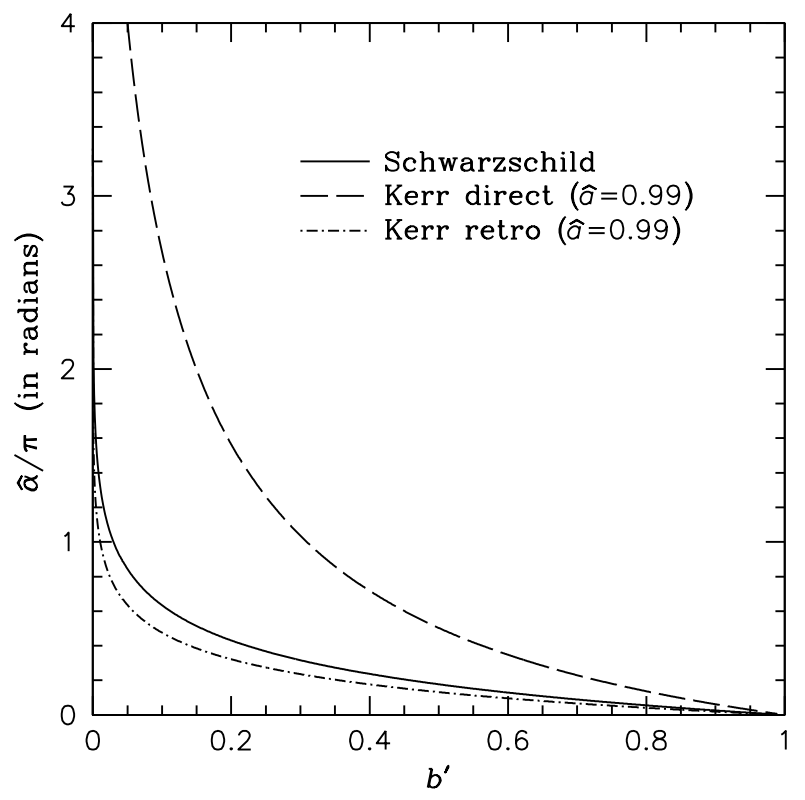

FIG. 5: Exact bending angle plotted as a function of the normalized impact parameter. The strong deflection regime is towards the left of this plot as $b^{\prime}$ goes to zero, and the weak deflection regime is as $b^{\prime} \rightarrow \infty$. The Schwarzschild bending angle is shown for reference.

"upstream" results in smaller bending angles. In both the direct and retro motion, as in the Schwarzschild case, the bending angle exceeds $2 \pi$, resulting in multiple loops and the formation of relativistic images. As a direct consequence of the difference between the direct and the retro side, as seen by an observer, these images will be offset to different amounts on either side.

A plot of the bending angle divided by $\pi$ as a function of $\hat{a}$ for $b^{\prime}=0.5$ is shown in Figure 6 to illustrate the dependence on the spin. The solid line in this plot is the zero-spin Schwarzschild case shown for reference.

\section{CONCLUSIONS}

We have presented a detailed calculation of the exact bending angle formula for a light ray in the equatorial plane of a Kerr black hole. The frame-dragging in the Kerr geometry has a different effect on the light ray depending on whether it is traversing with or against the spin direction. The extent to which it is deflected from its original path is much higher 


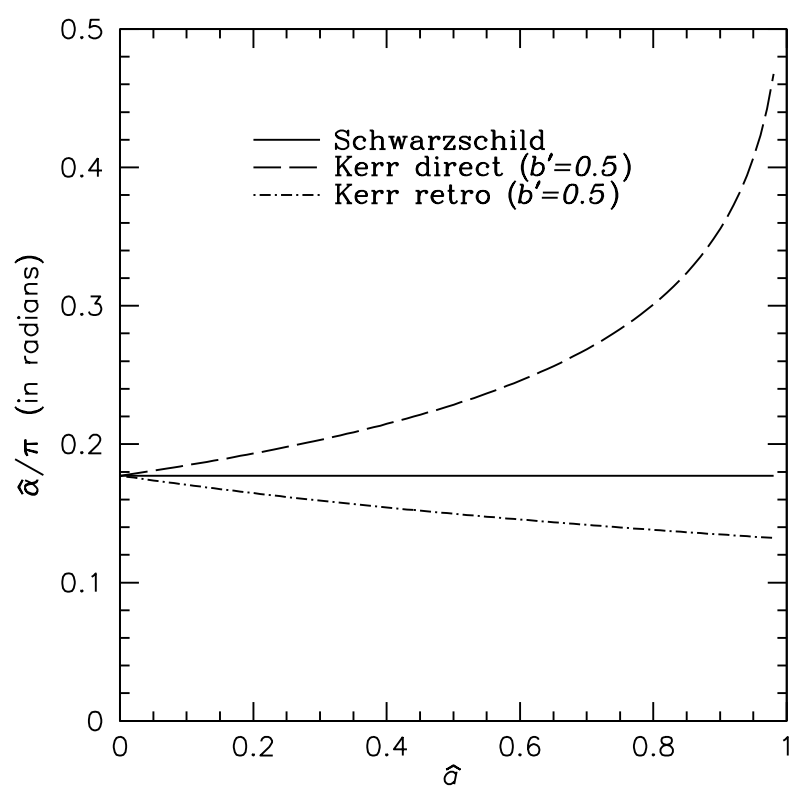

FIG. 6: Bending angle plotted as a function of spin for $b^{\prime}=0.5$. The Schwarzschild case is shown for reference.

for direct orbits than for retrograde orbits. As a result, relativistic images on the direct side shifts inward as $a$ increases, and outward on the retrograde side [9]. Higher order relativistic images can be studied by setting $\hat{\alpha}=2 n \pi$ and using the solution in the lens equation. In order to demonstrate this, however, we first need to determine the series expansions of the bending angle in both the strong and weak deflection limit. We present these two series expansions in a companion paper [9]. A direct application of this to the case when the source, lens and observer are perfectly lined up is also presented in [9].

Analytical solutions and results derived from them are crucial to our understanding of lensing images in general, and the Kerr geometry in particular. Even with our results here being exact, we have only considered the equatorial Kerr case. Our goal is to work with the full off-plane null geodesics in a similar manner to derive analytical solutions for predicting the behavior of a light ray as it spirals its way around the Kerr black hole before emerging towards the observer. 


\section{Acknowledgments}

S. V. I. thanks Arlie O. Petters for numerous helpful discussions. E. C. H. was funded by the Dr. Jerry D. Reber Student/Faculty Research Fund at SUNY Geneseo. The authors thank Kevin Cassidy for his support of this research fund.

[1] C. Darwin, Proc. R. Soc. London A249, 180 (1958); A263, 39 (1958).

[2] R. D. Atkinson, Astron. J. 70, 517 (1965).

[3] J.-P. Luminet, Astron. Astrophys. 75, 228 (1979).

[4] H. Ohanian, Am. J. Physics 55, 428 (1987).

[5] Misner, C., Thorne, K., \& Wheeler, J., Gravitation, W. H. Freeman and Company, San Francisco (1973).

[6] R. H. Boyer, R. W. Lindquist, J. Math. Phys, 8, (No. 2), 265 (1967).

[7] S. Chandrasekhar, The Mathematical Theory of Black Holes, (Oxford, 1992).

[8] S. V. Iyer and A. O. Petters, General Relativity and Gravitation 39, 1563-1582 (2007).

[9] S. V. Iyer and E. C. Hansen, "Strong and Weak Deflection of Light in the Equatorial Plane of a Kerr Black Hole," in preparation.

[10] P. F. Byrd and M. D. Friedman, Handbook of Elliptic Integrals for Engineers and Scientists, (Springer-Verlag, 1971). 